\title{
Implementación de NTS-TS 002 a pequeños y medianos hoteles. Caso Mola Hostel
}

\section{Implementation of NTS-TS 002 and its Adaptation to Small and Medium Hotels. Mola Hostel Case}

\author{
Laura Escobar Chiquiza ${ }^{1}$ \\ Nicolás Steven Castillo Tambo ${ }^{2}$ \\ Lida Esperanza Ruiz Martínez ${ }^{3}$
}

\section{OPEN ACCESS}

\section{(c) (1) $\Theta \Theta$}

\section{Copyright:}

(C2020. La revista Ingenierías USBmed proporciona acceso abierto a todos sus contenidos bajo los términos de la licencia creative commons Atribución no comercial SinDerivar 4.0 Internacional (CC BY-NC-ND 4.0)

Tipo de artículo: Investigación.

Recibido: 16-10-2019.

Revisado: 06-03-2020.

Aprobado: 05-05-2020.

Doi: $10.21500 / 20275846.4359$

\section{Referenciar así:}

L. Escobar-Chiquiza, N. S. CastilloTambo and L.E. Ruiz-Martínez, "Implementación de NTS-TS 002 a pequeños y medianos hoteles. Caso Mola Hostel," Ingenierías USBMed, vol. 11, no. 2, pp. 2-12, 2020 .

\section{Disponibilidad de datos:}

todos los datos relevantes están dentro del artículo, así como los archivos de soporte de información.

\section{Conflicto de intereses:}

los autores han declarado que no hay conflicto de intereses.

Editor: Andrés Felipe Hernández. Universidad de San Buenaventura, Medellín, Colombia.

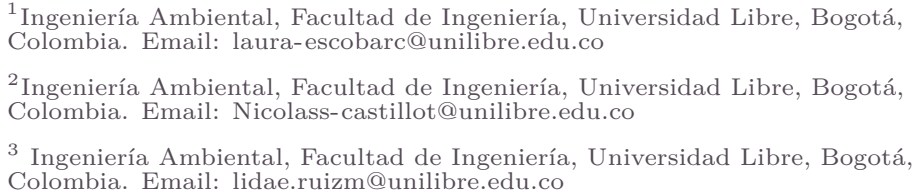

Resumen. La industria hotelera es uno de los sectores más importantes a nivel internacional debido a que contribuye al crecimiento económico de cada país. Además, es uno de los sectores que genera gran impacto ambiental, dado el consumo y aprovechamiento de los recursos naturales para la operación de los mismos. Para mitigar estos impactos existen normas a nivel mundial como la Green Globe 21, Green Key, ISO 14001 y en el caso de Colombia la NTS-TS 002, que presenta una disparidad por su aplicación a grandes hoteles, excluyendo a los de menor tamaño. Por esta razón, el presente artículo busca analizar los lineamientos metodológicos de la norma técnica sectorial NTS-TS 002 en el establecimiento hotelero Mola Hostel, mediante la implementación y aplicación de herramientas como la autoevaluación, la recolección de información primaria y secundaria, la formulación y aplicación de programas sostenibles, los eco balances y el análisis de encuestas en la escala de Likert. Esto permiten identificar las principales dificultades para la aplicación de la norma técnica en los pequeños y medianos hoteles y refleja cómo los esfuerzos en la adaptabilidad consiguen una mitigación de impactos ambientales significativa, reduciendo el consumo de energía, agua y residuos dentro del establecimiento hotelero objeto de estudio.

Palabras Clave. NTS-TS 002, Establecimiento de Alojamiento y Hospedaje (EAH), Impacto Ambiental, Eco-balances, Escala de Likert, Indicador Ambiental.

Abstract. The hotel industry is one of the most important sectors internationally, because it contributes to the economic growth of each country. Also, is one of the sectors that generates a considerable environmental impact, given the consumption and use of natural resources for the operation thereof. To mitigate these impacts, there are worldwide standards such as the Green Globe 21, Green Key, ISO 14001 and in the case of Colombia the NTS-TS 002, which presents a disparity due to its application to large hotels, excluding smaller ones. For this reason, this article seeks to analyze the methodological guidelines of the sectoral technical norm NTS-TS 002 in the Mola Hostel hotel establishment, through the implementation and application of tools such as self-evaluation, collection of primary and secondary information, formulation and application of programs sustainable, eco-balances and analysis of surveys on the Likert scale. These allow identifying the main difficulties for the application of the technical standard in small and medium-sized hotels and reflect how the efforts in the adaptability, achieve a mitigation of significant environmental impacts, reducing the consumption of energy, water and waste within the hotel establishment under study.

Keywords. NTS-TS 002, Establishment of Accommodation and Lodging (EAH), Environmental Impact, Eco-balances, Likert Scale, Environmental Indicator. 


\section{Introducción}

América Latina se encuentra en el tercer lugar mundialmente en recibir el mayor número de turistas, estando Colombia en el quinto lugar de los países con mayor flujo migratorio y una representación del $0.23 \%$, estimando 4.11 millones de migraciones para el año 2017 [1]. Esto gracias a la ubicación geográfica que posee, pues es el único país que cuenta con dos costas, tanto en el océano Pacífico como en el Atlántico, y una variedad en el relieve montañoso que permite tener diferentes pisos climáticos, que van desde cálido hasta páramo, haciendo del país un atractivo turístico para el mundo entero [2].

Este flujo migratorio no es más que el reflejo de la importancia e impacto económico que tiene el sector turístico en Colombia, y no es para menos, ya que, según el Ministerio de Comercio, Industria y Turismo en un informe realizado en el año 2017 junto con el Consejo Mundial de Viajes y Turismo, se estimó un crecimiento del $3.2 \%$ para el año 2018, representando aproximadamente un ingreso de 55,1 billones de pesos en la economía colombiana [3].

En consecuencia con lo anterior, el sector hotelero en Colombia ha tenido un incremento exponencial justificado en el ingreso de turistas al país. Esto es una gran oportunidad de negocio, ya que como lo publicó el DANE en un informe sobre la media mensual de los hoteles, los ingresos reales de los establecimientos hoteleros para el año 2018 crecieron en un $7.6 \%$ en comparación con el año 2017 [4].

Sin embargo, se debe tener en cuenta su clasificación de acuerdo a los servicios ofrecidos y a su infraestructura. En el caso de Colombia se tiene la Clasificación Industrial Internacional Uniforme (CIIU), allí se encuentran establecimientos tales como: albergues, aparta-hoteles, alojamientos rurales, viviendas turísticas, hoteles, presentes en mayor cantidad, y hostales [5].

Dada la clasificación anteriormente mencionada, en lo referente a los hostales se evidenció un crecimiento significativo, pues para el año 2012 representaban el $7.3 \%$ del sector hotelero en el país y para el año 2017 eran el $8.3 \%$ de establecimientos registrados en el Registro Nacional de Turismo (RNT) con un total de 1.366 hostales [6]. Para el caso de la ciudad de Bogotá, los hostales representaron el 8.5\% del sector hotelero, siendo el segundo porcentaje más alto luego de los hoteles registrados en el RNT [6]. Por lo tanto, este tipo de establecimientos es un objeto de estudio apropiado para la presente investigación.

A. Las consecuencias ambientales del sector hotelero El crecimiento del sector turístico y hotelero sumado a los altos estándares de calidad de los establecimientos para los turistas, además de la alta demanda de recursos que estos imponen, ha venido generando cuestionamientos referentes a los impactos negativos en el medio ambiente que puede dar como resultado la actividad hotelera [7].

Autores como Vargas et al. [8] señalan que estos sectores son sensibles al medio ambiente debido a que el consumo y/o aprovechamiento de recursos naturales es el sustento de su actividad. Sin embargo, el deterioro de los mismos es evidente. Por ejemplo, el gasto de agua promedio de un turista en España es de 0.80 $\mathrm{m}^{3}$, mientras que el de un español es de $0.25 \mathrm{~m}^{3}$, es decir, $0.55 \mathrm{~m}^{3}$ de agua más realizando las mismas actividades [9]. Esto evidencia un incremento significativo en el consumo de agua por parte de los establecimientos de hospedaje.

Consiguiente a esto, Colombia, en lo transcurrido del año 2015, generó un vertimiento total de 10508.464 $\mathrm{m}^{3}$ y una generación total de residuos sólidos convencionales de $22430985 \mathrm{~kg} /$ año, además de una disminución en la inversión del manejo de residuos sólidos y del recurso hídrico con variaciones negativas de $28.7 \%$ y $88 \%$, respectivamente, por parte de los establecimientos de alojamiento y hospedaje. En el caso de Bogotá estos vertimientos fueron de $1292583 \mathrm{~m}^{3}$ de agua y una generación de 4838133 kilogramos de residuos sólidos para el mismo año [10].

B. Certificaciones en el sector hotelero

Individualmente las empresas del sector turístico no son consideradas como causantes de grandes daños ambientales, pero la suma de las pequeñas acciones son las que conllevan a que las cifras anteriormente mencionadas sean una señal de alerta para el sector [11].

Esto conlleva a que la industria busque y adopte medidas de prevención y mitigación de los impactos ambientales que puedan producir por sus actividades de operación. Para ello en la actualidad existen diversas certificaciones en el sector hotelero que buscan la mitigación de los impactos y además generar sostenibilidad por parte de los EAH, a través de la aplicación de la gestión ambiental. Dentro de estas certificaciones se encuentra Green Globe 21, Green Key, ISO 9001 e ISO 14001 a nivel internacional. Para el caso de Colombia se cuenta con la norma técnica sectorial NTS-TS 002, creada por el Ministerio de Industria y Turismo en el año de 2006 con el objetivo de desarrollar programas de sostenibilidad basados en tres principales ejes: ambiental, sociocultural y económico. Aquello permite atender al deseo de los turistas de hospedarse en hoteles que respetan los parámetros ambientales, buscando un equilibrio sostenible y generando calidad turística en el país [12].

Estas respuestas, aunque parecen ser un camino viable para combatir todas las problemáticas, además de crear un incentivo a los EAH, no dejan de ser la solución a las afectaciones por parte de los mismos; esto debido a que gran parte de las normativas, en especial la NTSTS 002, se elaboraron pensando en los grandes estableci- 
mientos hoteleros, dificultando su implementación en los pequeños y medianos hoteles y hostales del país [13].

A nivel nacional para el año 2015, en una encuesta realizada por la Departamento Administrativo Nacional de Estadísticas, la cantidad de hoteles con certificación ambiental en la norma técnica sectorial era de tan solo 65 otorgadas y 106 en fase de implementación, siendo esta cifra muy baja en comparación a la cantidad de hoteles registrados para esa fecha [10].

Para la ciudad de Bogotá el panorama tampoco es alentador, ya que en un censo realizado a 558 establecimientos de alojamiento y hospedaje para el año de 2018, por parte del Instituto Distrital de Turismo, el resultado fue que la gran mayoría de la oferta de alojamiento en la ciudad está concentrada en establecimientos de pequeña y mediana envergadura, representando el $81 \%$ del sector hotelero en la capital. Adicional a esto, tan solo 105 establecimientos de los 558 han llevado a cabo algún programa referente a la NTSSostenibilidad [14].

Los bajos estándares de implementación de la norma técnica sectorial se relacionan con el hecho de que al momento de realizar la autoevaluación requerida se observa que muchos de los criterios no son aplicables para sus establecimientos o que implicarían un gasto económico considerable para su implementación. Además, se tiene el cambio de la normativa sectorial, que pasa de ser optativa a obligatoria de acuerdo con el artículo 5 de la Ley 1558 de 2012, donde se recalca el desarrollo, promoción y competitividad del sector turístico por medio de mecanismos enfocados en la conservación, protección y aprovechamiento de los recursos naturales en Colombia [15].

Por otra parte, se encuentra lo dispuesto en la Resolución 3860 del año 2015, la cual da como plazo máximo para la implementación de la norma técnica sectorial el mes de marzo del año 2017, fecha en que los prestadores de servicios turísticos debían demostrar el cumplimiento de las normas técnicas sectoriales, según sea el tipo de actividad y así obtener el registro único nacional (RNT) [16].

Esta obligatoriedad genera mayor incertidumbre, inconsistencia y conflicto para aquellos establecimientos hoteleros de menor tamaño, afectándolos de manera directa, puesto que tal y como lo asegura el Sr. Salamanca, presidente del gremio hotelero del Tolima, en entrevista con el periódico Nuevo Día: "Muchos establecimientos no cuentan con los recursos necesarios para pagar las auditorías ni hacer las inversiones necesarias para dar cumplimiento a los criterios requeridos por la norma y poder lograr su certificación" [13]. A esto se le suma el estudio elaborado por Aldana [17], quien al realizar una serie de encuestas a los hoteles certificados por esta norma en la ciudad de Bogotá evidenció que, aunque poseen una certificación alta en sostenibilidad, han tenido una serie de dificultades en su implementación debido a la falta de conocimientos, presupuesto y en ocasiones la incertidumbre de saber si los programas y acciones empleados por los EAH han tenido un cambio significativo en el medio ambiente.

No obstante, existen organizaciones con responsabilidad social y ambiental que quieren incorporar dentro de sus prácticas la NTS-TS 002 con el fin de minimizar los impactos que puedan producir al medio ambiente, como es el caso de estudio del EAH Mola Hostel, ubicado en Bogotá en la localidad de La Candelaria, que se caracteriza por ser un atractivo turístico de tipo histórico-cultural, concentrando el $70 \%$ de los atractivos de la ciudad [18].

Este EAH caso de estudio es uno de los hostales donde se observaron, por medio de la autoevaluación, las mismas dificultades e irregularidades a la hora de dar cumplimiento a los criterios descritos en la norma referentes al eje ambiental, económico y cultural. Esta situación imposibilita el cumplimiento del cien por ciento de la misma, tal y como lo exige la norma. Sin embargo, adaptando y modificando paulatinamente los criterios requeridos se puede garantizar la sostenibilidad dentro del establecimiento de alojamiento y hospedaje.

Además de esto, Mola Hostel abre la posibilidad de debatir sobre la necesidad de evaluar y analizar la norma NTS-TS 002 para este tipo de establecimientos y cuestionar si es posible su implementación en pequeños hoteles y hostales tal y como se presenta en la norma o si por el contrario es necesaria la creación de manuales adaptados a este tipo de EAH.

\section{Desarrollo del artículo}

La metodología llevada a cabo en el presente artículo se desarrolla en dos partes. La primera parte consta de la descripción del área de estudio donde se va a elaborar e implementar la norma técnica sectorial NTS-TS 002. La segunda parte se divide en tres fases metodológicas que se describen a continuación:

\section{A. Fases metodológicas}

La metodología llevada a cabo para la implementación y adaptación de la norma técnica sectorial NTS-TS 002 en el establecimiento hotelero Mola Hostel tuvo tres fases, que a su vez contienen los principales pasos, con el fin de alcanzar los objetivos propuestos (Figura 1).

1) Fase 1: análisis situacional

- Área de estudio: el reconocimiento del área de estudio se llevó a cabo de forma general y especifica. La parte general consistió en la identificación de la localización donde se encuentra ubicado el EAH y, en cuanto a la forma específica, se identificaron aspectos tales como: el tipo de infraestructura del establecimiento hotelero, la capacidad, las áreas comunes y habitaciones, entre otros.

- Autoevaluación: de acuerdo a la guía metodológica propuesta por el Ministerio, uno de los primeros 


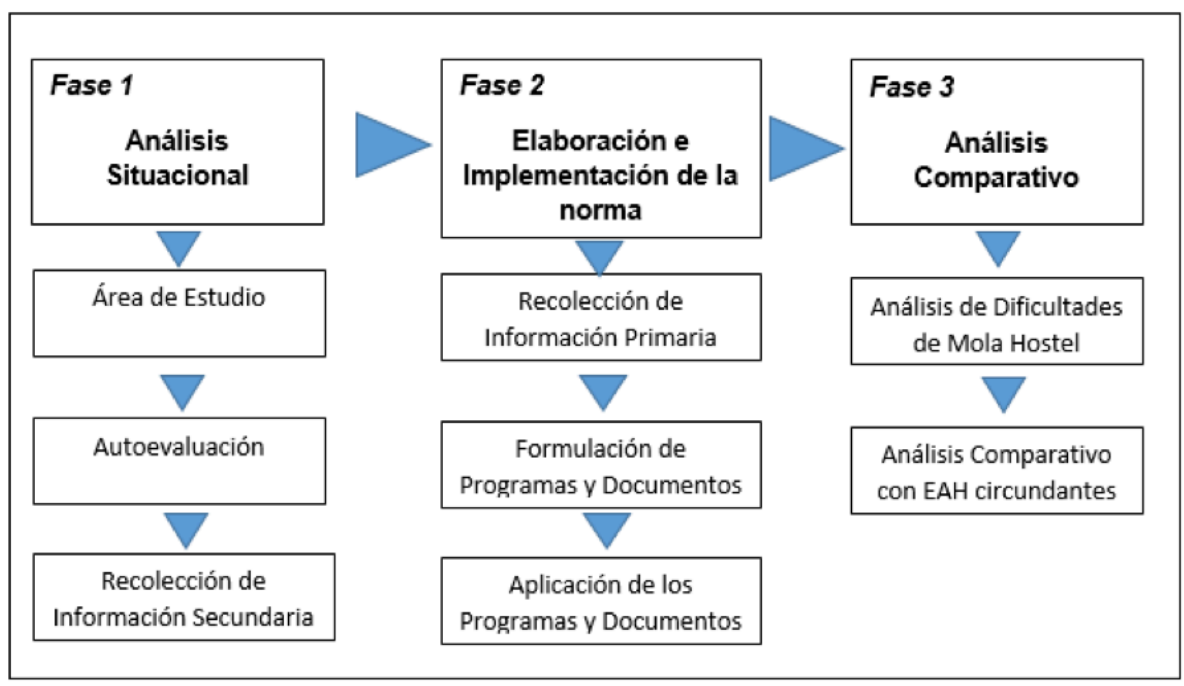

Figura 1. Fases de la metodología

pasos es la realización de la autoevaluación, que consiste en llenar una lista de chequeo como declaración de primera parte del proveedor del servicio de acuerdo con la NTC ISO- IEC 17050. Esta se divide en la parte de gestión de sostenibilidad ambiental, sociocultural y económica. Fue aplicada al EAH Mola Hostel, verificando los requisitos de cumplimiento y obteniendo como resultado una tabla que permitió identificar aquellos aspectos que se estaban llevando a cabalidad, además de priorizar los requisitos que se cumplieron de forma parcial o de forma incompleta.

- Recolección de información secundaria: la recolección de información secundaria se basó en el recaudo de casos de éxitos en la implementación de la NTS-TS 002 aplicados a los establecimientos hoteleros en Colombia. Además, se evaluaron diferentes alternativas de sistemas de gestión ambiental y producción más limpia, aplicables al sector hotelero de acuerdo a la metodología propuesta por el Centro de Actividad Regional para la Producción más Limpia [19] y por Medina \& Zea [20].

Por otro lado, se buscó dar cumplimiento a los lineamientos generales en el desarrollo del marco legal y normativo para el caso de Mola Hostel, por medio de la exploración de normativas aplicables y vigentes al establecimiento hotelero en el ámbito ambiental, turístico, de patrimonio cultural, social, laboral y de seguridad, partiendo de fuentes de información como la constitución, el Congreso, ministerios, entre otros.

2) Fase 2: elaboración e implementación de la norma - Recolección de información primaria: la recolección de información primaria correspondiente al establecimiento hotelero intervenido se realizó de acuerdo a la metodología propuesta por la norma ISO 14031 de 2015 [21], [22] y la guía metodológica de la norma técnica sectorial, que consiste en el uso de herramientas como ecomapas, diagramas de procesos y matrices de eva- luación de impactos. Esto permite la identificación de las zonas del EAH que producen un consumo de agua, energía y residuos ordinarios y peligrosos, para posteriormente obtener la caracterización del desempeño ambiental de los indicadores de acuerdo al número de huéspedes por mes en un lapso de tiempo de tres meses, desde el mes de abril de 2018 hasta el mes de junio del mismo año y posteriormente.

- Formulación de programas y documentos: la formulación y elaboración de los programas y documentos se realizó de acuerdo a la guía metodológica dispuesta por el Ministerio de Comercio, Industria y Turismo de Colombia, donde se enfatizó en el eje ambiental, teniendo como finalidad la implementación de estrategias encaminadas a la correcta gestión en el ahorro y el uso racional de los recursos naturales que demanda el establecimiento Mola Hostel. Esto con el objetivo de minimizar los impactos ambientales y dar cumplimiento a la normativa vigente, sin dejar de lado los ámbitos socioculturales y económicos.

-Aplicación de los programas y documentos: de acuerdo a lo planteado en la formulación de los programas y documentos del eje ambiental, sociocultural y económico se llevó a cabo su ejecución percatándose en el cumplimiento de los objetivos propuestos en cada uno de los programas, para posteriormente realizar un análisis comparativo por medio del uso de herramientas como las gráficas de barras, determinando la evolución del antes y el después de su aplicación.

3) Fase 3: análisis comparativo

- Análisis de dificultades de Mola Hostel: luego de la elaboración e implementación de la NTS-TS 002 en el EAH Mola Hostel se procedió a analizar los requisitos que presentaron algún tipo de problemática o dificultad a la hora de su implementación de acuerdo a lo exigido por la norma, evaluando las posibles causas de las dificultades. 

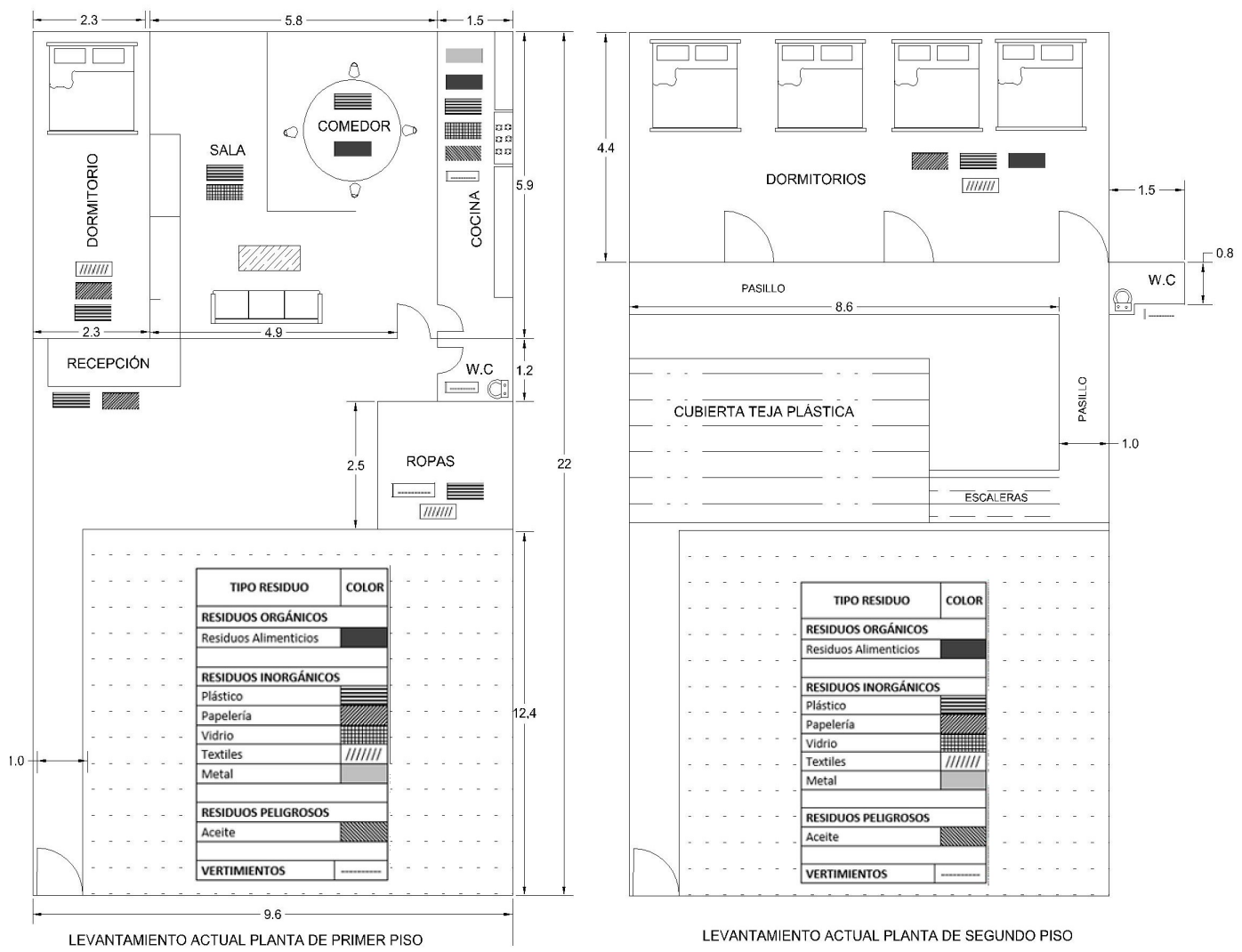

Figura 2. Levantamiento del ecopama Mola Hostel

- Análisis comparativo con hoteles circundantes: el análisis comparativo se llevó a cabo por medio de la metodología propuesta por Chan [23], en donde se hace uso de la escala de Likert. Para el presente artículo la muestra tomada fue de 14 hoteles y hostales aledaños a Mola Hostel, donde inicialmente se realizaron 2 preguntas sobre el conocimiento y la elaboración de la norma técnica sectorial en los establecimientos. La afirmación de esta pregunta delimita 9 afirmaciones posteriores sobre la experiencia tenida a la hora de elaborar e implementar la norma técnica sectorial. Esta información se representa en los resultados.

El método de evaluación de cada pregunta se representa de la siguiente forma:

Tabla 1. Criterios de evaluación.

\begin{tabular}{|lc|}
\hline \hline Criterio & Valor \\
\hline \hline Totalmente en desacuerdo & 1 \\
En desacuerdo & 2 \\
Ni de acuerdo ni en desacuerdo & 3 \\
De acuerdo & 4 \\
Totalmente de acuerdo & 5 \\
\hline
\end{tabular}

Fuente: Autores, 2019.

\section{Resultados}

A. Fase 1: análisis situacional

- Área de estudio: de forma general Mola Hostel se encuentra ubicado en la localidad número diecisiete correspondiente a La Candelaria en el centro de la ciudad de Bogotá, que cuenta con una extensión de 183.89 hectáreas con coordenadas geográficas $\mathrm{N} 4^{\circ} 35^{\prime \prime} 30^{\prime}$ y O $74^{\circ}$ 04" 27', más exactamente en la calle 12d\#1 ${ }^{\mathrm{a}}-04$ [24].

Esta localidad limita al norte con la calle 13 y la carrera 2a, al sur con la calle $12 \mathrm{~b}$, al este con la avenida Circunvalar o carrera $5 \mathrm{a}$ y al oeste con la carrera 10. Además, es la más pequeña de Bogotá y está inmersa dentro de la localidad de Santafé (3), limitando también con Los Mártires (14), San Cristóbal (4) y Chapinero (2) [25].

En los aspectos específicos Mola Hostel es una apuesta netamente colombiana, que nace con la intención de fomentar el conocimiento sobre el pueblo indígena Guna y su tradicional arte Mola, elaborado cuidadosamente por las mujeres pertenecientes a la tribu. A nivel de infraestructura el establecimiento hotelero posee un espacio dispuesto por un área compartida de 7 camarotes ubicados en la planta del segundo piso. Tiene un total de 14 camas individuales con conexión e iluminación individual y una habitación privada con cama King Size para dos personas, además de contar con un 
Tabla 2. Autoevaluación.

\begin{tabular}{|c|c|c|c|c|}
\hline & No cumplía & No aplica & $\begin{array}{l}\text { No se adapta } \\
\text { parcialmente }\end{array}$ & $\begin{array}{c}\% \text { Incumplimiento } \\
\text { total }\end{array}$ \\
\hline Gestión de sostenibilidad & $40 \%$ & $0 \%$ & $0 \%$ & $6.52 \%$ \\
\hline Requisitos ambientales & $21.74 \%$ & $8.7 \%$ & $15.21 \%$ & $22.82 \%$ \\
\hline Requisitos socio-culturales & $21 \%$ & $15 \%$ & $0 \%$ & $7.6 \%$ \\
\hline Requisitos económicos & $22.2 \%$ & $0 \%$ & $0 \%$ & $2.17 \%$ \\
\hline Norma NT-TS 002 & $26 \%$ & $7.6 \%$ & $5.43 \%$ & $39.11 \%$ \\
\hline
\end{tabular}

Fuente: Autores, 2019.

área social donde los huéspedes tienen a su disposición un lugar confortable para compartir, que cuenta con televisión, barra-comedor y mobiliario. El establecimiento hotelero dispone de una cocina, así como de área de lavandería con secadora. Esto se puede observar en el levantamiento del ecomapa del hostal (Figura 2).

- Autoevaluación: para los resultados de la autoevaluación que se le realizó al EAH Mola Hostel, de acuerdo al porcentaje de incumplimiento, se obtuvo la Tabla 2.

B. Fase 2: elaboración e implementación de la norma - Recolección de información primaria: de acuerdo a la metodología empleada, primero se obtuvo el levantamiento del ecomapa (Figura 2), donde se plasman los residuos y vertimientos generados por los procesos que se llevan a cabo en las diferentes zonas del establecimiento hotelero Mola Hostel.

Por otro lado, el resultado de la caracterización de los indicadores correspondientes a los consumos de agua, energía y generación de residuos se muestra en las Tablas 3, 4 y 5 .

Tabla 3. Indicador de consumo de energía.

\begin{tabular}{|lccc|}
\hline \hline Mes & Kw & $\begin{array}{c}\text { Número de } \\
\text { huéspedes }\end{array}$ & $\begin{array}{c}\text { Kw/Huésped } \\
\text { mes }\end{array}$ \\
\hline \hline Abril & 105 & 8 & 13.125 \\
Mayo & 256 & 40 & 6.40 \\
Junio & 290 & 55 & 5.27 \\
\hline
\end{tabular}

Fuente: Autores, 2019.

Tabla 4. Indicador de consumo de agua.

\begin{tabular}{|lccc|}
\hline \hline Bimensual & $\mathbf{m}^{3}$ & $\begin{array}{c}\text { Número de } \\
\text { huéspedes }\end{array}$ & $\begin{array}{c}\mathbf{m}^{3} \text { /Huésped- } \\
\text { bimensual }\end{array}$ \\
\hline \hline Febrero-Abril & 8 & 8 & 1.00 \\
Abril-Junio & 23 & 95 & 0.24 \\
\hline
\end{tabular}

Fuente: Autores, 2019.

Tabla 5. Indicador de generación de residuos.

\begin{tabular}{|lcc|}
\hline \hline Mes & $\begin{array}{c}\text { Residuos } \\
\text { ordinarios } \mathbf{( K g})\end{array}$ & $\begin{array}{c}\text { Residuos } \\
\text { peligrosos } \mathbf{( K g})\end{array}$ \\
\hline \hline Abril & 23 & 1 \\
Mayo & 31 & 0.5 \\
Junio & 22 & 0.5 \\
\hline
\end{tabular}

Fuente: Autores, 2019.
De acuerdo a la tabla anterior se realizó el método de cuarteo y estandarización, identificando el porcentaje de residuos generados en Mola Hostel como se observa en las Figuras 3 y 4.

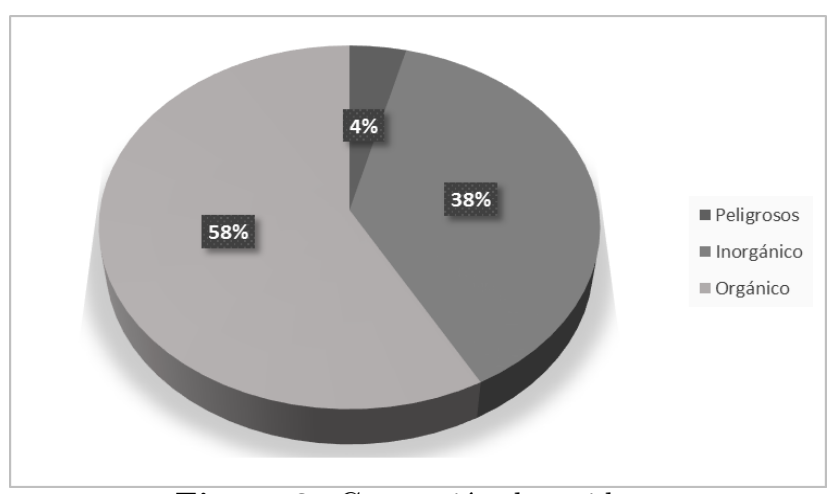

Figura 3. Generación de residuos

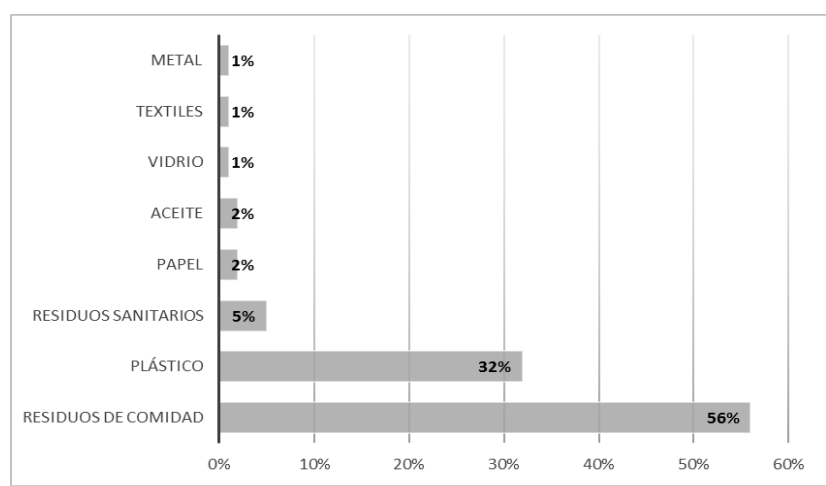

Figura 4. Clasificación de residuos

- Formulación de programas y documentos: se formularon programas para el ahorro y uso eficiente de agua, energía, residuos, entre otros. Esto de acuerdo a los requisitos dispuestos por la NTS-TS 002, con la finalidad de crear metas y acciones encaminadas a la correcta gestión de los recursos, las diferentes formas de ahorro, mitigación del impacto ambiental y disposición final, disminuyendo el consumo de los recursos que se lleva a cabo en las instalaciones de Mola Hostel para dar cumplimiento a la normativa vigente.

- Aplicación de los programas y documentos: Luego de la formulación de los programas se procedió a su implementación. Se verificó el comportamiento y la evo- 
Tabla 6. Requisitos ambientales no aplicables al EAH NTS-TS 002.

\begin{tabular}{|c|c|c|c|c|}
\hline Componente & \begin{tabular}{|c|}
$\mathbf{N}^{\circ}$ \\
Requisito
\end{tabular} & $\begin{array}{c}\text { Descripción del } \\
\text { requisito }\end{array}$ & $\begin{array}{c}\text { Causa del } \\
\text { incumplimiento }\end{array}$ & Adaptación del requisito \\
\hline \multirow{4}{*}{$\begin{array}{l}\text { Apoyo a } \\
\text { programas de } \\
\text { protección y } \\
\text { uso sostenible }\end{array}$} & 21 & $\begin{array}{l}\text { Respetar las rondas hídri- } \\
\text { cas de los cuerpos de agua, } \\
\text { humedales, lagos, lagunas, } \\
\text { ciénagas y manglares, re- } \\
\text { servas de fauna y flora y á- } \\
\text { reas de alta amenaza y } \\
\text { riesgo no mitigable, de } \\
\text { acuerdo con la legislación } \\
\text { vigente aplicable. }\end{array}$ & $\begin{array}{l}\text { El EAH se encuentra lo- } \\
\text { calizado en la zona urbana } \\
\text { de Bogotá, por lo cual no } \\
\text { cuenta con la presencia de } \\
\text { rondas hídricas cercanas o } \\
\text { reservas de flora y fauna. }\end{array}$ & $\begin{array}{l}\text { Se recomienda que la norma sectorial } \\
\text { en los EAH ubicados en zonas urbanas } \\
\text { y para aquellos que no apliquen, se } \\
\text { enfoque en capacitaciones o campañas } \\
\text { tanto para personal del establecimiento, } \\
\text { como para los huéspedes, resaltando } \\
\text { la importancia de las rondas hídricas y } \\
\text { reservas de flora y fauna más cercanas. }\end{array}$ \\
\hline & 23 & $\begin{array}{l}\text { Contar con información } \\
\text { que identifique con su } \\
\text { nombre local y científico } \\
\text { las especies arbustivas y ar- } \\
\text { bóreas más representativas } \\
\text { de su establecimiento. }\end{array}$ & $\begin{array}{l}\text { Por encontrarse el EAH } \\
\text { en una zona urbanística, } \\
\text { sumado al tipo de in- } \\
\text { fraestructura del EAH, no } \\
\text { cuenta o podría contar } \\
\text { con algún tipo de especies } \\
\text { arbustivas o arbóreas. }\end{array}$ & $\begin{array}{l}\text { Para aquellos EAH en los que no } \\
\text { aplique el siguiente requisito se re- } \\
\text { comienda el apadrinamiento de una } \\
\text { zona verde cercana a las instalaciones. }\end{array}$ \\
\hline & 24 & $\begin{array}{l}\text { Adelantar acciones para } \\
\text { que su iluminación externa } \\
\text { no cause alteraciones en el } \\
\text { medio natural. }\end{array}$ & $\begin{array}{l}\text { No se cuenta con algún } \\
\text { tipo de medio natural en } \\
\text { los alrededores del EAH. } \\
\text { Además, la iluminación ex- } \\
\text { terior se encuentra a cargo } \\
\text { de la empresa Codensa. }\end{array}$ & $\begin{array}{l}\text { Se recomienda que el presente numeral } \\
\text { se relacione con los requisitos dis- } \\
\text { puestos en la gestión de energía. Aquí } \\
\text { se propone el cambio de las luminarias } \\
\text { que puedan afectar el entorno natural. }\end{array}$ \\
\hline & 25 & $\begin{array}{l}\text { Adelantar acciones para } \\
\text { que en la cobertura de las } \\
\text { áreas verdes y su orna- } \\
\text { mentación prevalezcan las } \\
\text { especies nativas del destino. }\end{array}$ & $\begin{array}{l}\text { En la localización del } \\
\text { EAH no hay ningún tipo } \\
\text { de especie nativa debido a } \\
\text { la urbanización. }\end{array}$ & $\begin{array}{l}\text { Para aquellos EAH que no cuenten } \\
\text { con áreas verdes se recomienda que } \\
\text { los establecimientos en un espacio } \\
\text { determinado fabriquen un jardín con } \\
\text { elementos reciclables donde se planten } \\
\text { especies representativas de la zona }\end{array}$ \\
\hline
\end{tabular}

Fuente: Autores, 2019.

lución por medio de los indicadores, teniendo en cuenta la cantidad de huéspedes que se hospedaron en el mes. Aparece entonces la siguiente información:

- Energía: el consumo de energía antes de la implementación tuvo un promedio de $8.26 \mathrm{Kw} /$ huésped-mes, luego de la implementación del programa se redujo a $2.48 \%$, como se puede observar en la Figura 5. La optimización y eficiencia fue del $70 \%$.

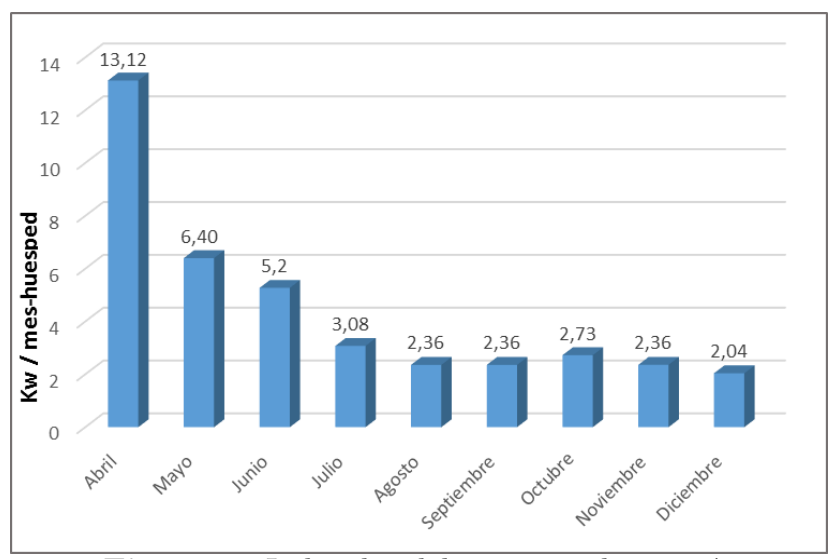

Figura 5. Indicador del consumo de energía
- Agua: el consumo de agua antes de la implementación de los programas tenía un promedio de $1 \mathrm{~m}^{3} /$ huésped-bimensual. Luego de la implementación del programa de ahorro y uso eficiente del agua el consumo se redujo a un promedio de $0,24 \mathrm{~m} 3 /$ huésped-bimensual. Esto se puede observar en la Figura 6, donde se tuvo una optimización y eficiencia del $76 \%$ aproximadamente.

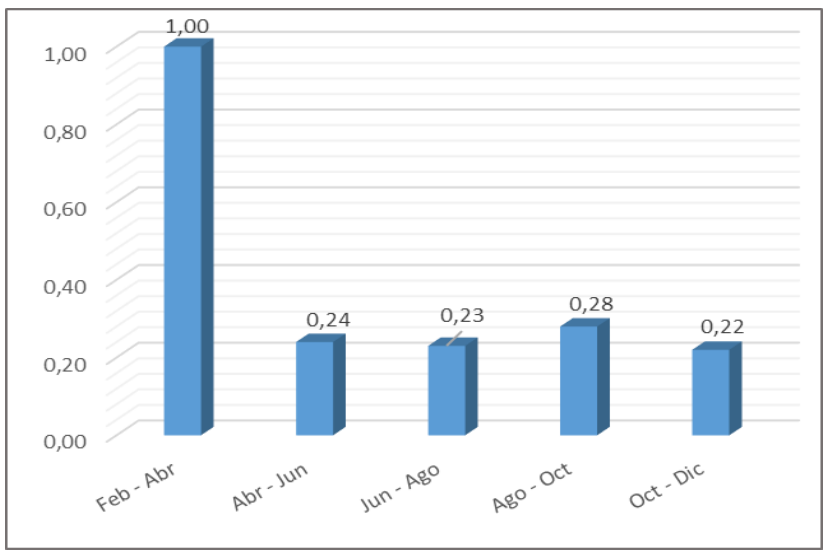

Figura 6. Indicador del consumo de agua 
Tabla 7. Requisitos ambientales no aplicables parcialmente al EAH.

\begin{tabular}{|c|c|c|c|c|}
\hline Componente & \begin{tabular}{c|}
$\mathbf{N}^{\circ}$ \\
Requisito
\end{tabular} & $\begin{array}{l}\text { Descripción del } \\
\text { requisito }\end{array}$ & $\begin{array}{c}\text { Causa del } \\
\text { incumplimiento }\end{array}$ & Adaptación del requisito \\
\hline GEI & 54 & $\begin{array}{l}\text { Identificar las fuentes de } \\
\text { emisión que generan GEI. }\end{array}$ & $\begin{array}{l}\text { Se presentaron dificultades } \\
\text { a la hora de disponer de un } \\
\text { documento estandarizado } \\
\text { por las autoridades ambi- } \\
\text { entales para estimar la hue- } \\
\text { lla de carbono producida } \\
\text { por los EAH en el país. }\end{array}$ & $\begin{array}{l}\text { Se recomienda que dentro de la norma } \\
\text { sectorial se estipule una metodología } \\
\text { estandarizada para la obtención de los } \\
\text { factores de conversión, con el fin de } \\
\text { realizar el correcto cálculo de la huella } \\
\text { de carbono. }\end{array}$ \\
\hline $\mathrm{SAO}$ & $\begin{array}{c}56,58,59 \\
60,61 \mathrm{y} \\
62\end{array}$ & $\begin{array}{l}\text { El componente de sus- } \\
\text { tancias agotadoras de } \\
\text { ozono (SAO), en los } \\
\text { requisitos ambientales, } \\
\text { está compuesto por el } \\
\text { registro, mantenimiento } \\
\text { y disposición final de los } \\
\text { sistemas de refrigeración y } \\
\text { climatización como lo es el } \\
\text { aire acondicionado. }\end{array}$ & $\begin{array}{l}\text { Dadas las condiciones } \\
\text { climáticas de Bogotá, los } \\
\text { equipos de climatización } \\
\text { como aires acondicionados } \\
\text { no son de alto uso. Como } \\
\text { se observó en el EAH } \\
\text { objeto de estudio no se } \\
\text { contaba con la presencia } \\
\text { de estos equipos, sino con } \\
\text { un equipo de refrigeración } \\
\text { (nevera), haciendo que la } \\
\text { generación de sustancias } \\
\text { agotadoras de la capa } \\
\text { de ozono (SAO) fuese } \\
\text { mínima dentro del EAH. }\end{array}$ & $\begin{array}{l}\text { Se recomienda incorporar para los } \\
\text { pequeños y medianos EAH que no } \\
\text { cuenten con más dos equipos de refrig- } \\
\text { eración y climatización. Asimismo, se } \\
\text { sugiere unificar el componente SAO } \\
\text { en un solo ítem que corresponda al } \\
\text { mantenimiento periódico y la correcta } \\
\text { disposición del gas refrigerante. } \\
\text { Adicionalmente, que para aquellos es- } \\
\text { tablecimientos que tengan equipos de } \\
\text { refrigeración con más de diez años de } \\
\text { vida se plantee como meta a medi- } \\
\text { ano plazo realizar el cambio de estos } \\
\text { equipos por unos libres de CFC. }\end{array}$ \\
\hline
\end{tabular}

Fuente: Autores, 2019.

- Residuos: en la generación de residuos ordinarios y peligrosos que se producían mensualmente en el establecimiento hotelero Mola Hostel se presentó una reducción del 20\%. Este resultado se logró gracias a la concientización de huéspedes y funcionarios con respecto a la correcta separación en la fuente para poder retornar a la vida productiva aquellos residuos aprovechables. Adicionalmente, la disminución de residuos peligrosos se logró debido a las compras a granel de los productos de aseo.

\section{Fase 3: análisis comparativo}

- Análisis de dificultades de Mola Hostel: de acuerdo a la Tabla 2 "Autoevaluación" y respecto a los criterios que no aplican para el EAH Mola Hostel se procedió a identificar las posibles causas de las dificultades. Además, se planteó una solución frente a la problemática evaluada, como se puede observar en la Tabla 6 y 7 .

- Análisis comparativo con hoteles circundantes: de acuerdo a las encuestas realizadas en los establecimientos hoteleros aledaños a Mola Hostel se obtuvo que tan solo el $38.46 \%$ había implementado la norma sectorial, el $30.76 \%$ la conocía, pero no la había implementado y el $38.46 \%$ no tenían conocimiento de la existencia de la norma.

Ahora bien, los resultados de los establecimientos hoteleros que implementaron la norma (38.46\%) y que respondieron las 9 afirmaciones referentes a la experiencia vivida al momento de elaborar e implementar la normal se observan en las Tablas 8 y 9.
Tabla 8. Afirmaciones encuestas.

\begin{tabular}{|c|c|}
\hline Afirmación & Pregunta \\
\hline 1 & $\begin{array}{l}\text { La sostenibilidad es impor- } \\
\text { tante para el EAH. }\end{array}$ \\
\hline 2 & $\begin{array}{l}\text { Cuenta con los recursos físicos } \\
\text { y financieros para implemen- } \\
\text { tar la norma. }\end{array}$ \\
\hline 3 & $\begin{array}{l}\text { La información es adecuada y } \\
\text { fácil de comprender. }\end{array}$ \\
\hline 4 & $\begin{array}{l}\text { Los requisitos tuvieron un im- } \\
\text { pacto positivo. }\end{array}$ \\
\hline 5 & $\begin{array}{l}\text { Elaboró la totalidad de los } \\
\text { programas solicitados. }\end{array}$ \\
\hline 6 & $\begin{array}{l}\text { Redujo el consumo de agua, } \\
\text { energía y residuos. }\end{array}$ \\
\hline 7 & $\begin{array}{l}\text { Cumplió el } 100 \% \text { de los requi- } \\
\text { sitos solicitados. }\end{array}$ \\
\hline & Considera que deberían \\
\hline 8 & $\begin{array}{l}\text { realizarse adecuaciones a } \\
\text { la norma para tener un } \\
\text { mayor impacto en pequeños } \\
\text { y medianos hoteles. }\end{array}$ \\
\hline 9 & $\begin{array}{l}\text { Presentó dificultades o identi- } \\
\text { ficó requisitos que no aplica- } \\
\text { ban para su EAH. }\end{array}$ \\
\hline
\end{tabular}

Fuente: Autores, 2019.

En términos generales la aceptación de la norma en los establecimientos que la habían implementado tuvo un impacto positivo gracias a la reducción del consumo de agua, energía y residuos. Sin embargo, las 
Tabla 9. Resultado encuestas.

\begin{tabular}{|cccccc|}
\hline \hline Afirmación & Porcentaje obtenido & Puntaje Máximo & Puntaje Mínimo & Promedio \% de aceptación \\
\hline \hline 1 & 25 & 25 & 5 & 5 & $100 \%$ \\
2 & 22 & 25 & 5 & 4.4 & 3.8 \\
3 & 19 & 25 & 5 & 5 & $76 \%$ \\
4 & 25 & 25 & 5 & 5.4 & $100 \%$ \\
5 & 20 & 25 & 5 & 4.6 & $32 \%$ \\
6 & 23 & 25 & 5 & 4.6 & $60 \%$ \\
7 & 15 & 25 & 5 & 4.2 & $82 \%$ \\
5 & 23 & 25 & 5 & & $84 \%$ \\
\hline
\end{tabular}

Fuente: Autores, 2019.

afirmaciones 8 y 9 que mencionan dificultad a la hora de elaborar y realizar adecuaciones a la norma superaron el $80 \%$, lo que indica que alcanzar los impactos positivos no fue del todo fácil.

\section{Conclusiones y recomendaciones}

- El porcentaje de incumplimiento por parte del EAH fue de tan solo $39.11 \%$, donde los requisitos ambientales presentaron dificultades e irregularidades a la hora de la autoevaluación de cumplimiento e implementación, obteniendo un porcentaje del $22.82 \%$. Este corresponde a un $8.7 \%$ de los requisitos no aplicables y a un $15.21 \%$ de los requisitos que parcialmente no pueden ser implementados en Mola Hostel. Lo anterior refleja falta de acciones y metodologías por parte de las autoridades competentes para la adaptabilidad de la norma técnica sectorial NTS-TS 002 en este tipo de establecimientos. - Al igual que en el estudio de Ortiz y Olivares [26] las acciones y metodologías que fueron adaptadas y empleadas en la formulación y aplicación de los diferentes programas tuvieron un impacto positivo en aquellos procesos y actividades que se desarrollaron en el establecimiento hotelero Mola Hostel. Por ejemplo, optimizando el consumo de energía en un $70 \%$, pasando de un consumo promedio de $8.26 \mathrm{kw} /$ mes-huésped a un promedio de $2.48 \mathrm{kw} /$ mes-huésped; un $76 \%$ para el consumo de agua, que pasó de $1 \mathrm{~m} 3$ /huésped-bimensual a $0.24 \mathrm{~m}^{3} /$ huésped-bimensual y un $20 \%$ en la generación de residuos, abriendo la posibilidad de replicar la metodología aplicada para aquellos pequeños y medianos hoteles que quieran convertirse en establecimientos hoteleros autosostenibles o con impactos ambientales de menor incidencia.

- De acuerdo a la recolección de información primaria, el área donde se presenta mayor consumo de agua y generación de vertimientos es el cuarto de lavado. Por otro lado, aquella que genera mayor cantidad de residuos es el área de la cocina, encontrando una relación con los estudios realizados por Pirani y Arafat [21]. Por lo tanto, se recomienda que los EAH prioricen sus actividades en la identificación de los impactos ambi- entales en este tipo de áreas y se enfoquen en plantear acciones preventivas y correctivas para la minimización de los mismos.

- Las principales limitantes que se presentaron en los requisitos ambientales están relacionadas con los EAH localizados en zonas rurales y de gran envergadura, pues como se evidencia en la Tabla 6 los requisitos están enfocados en áreas verdes y el tipo de infraestructura donde se encuentra el estudio de caso es urbano.

Por otro lado, en los requisitos referentes a GEI y SAO (Tabla 7) el sesgo que se tiene para los EAH de menor envergadura se evidencia con mayor notoriedad. Esto es debido a que los equipos y aparatos que puedan llegar a tener este tipo establecimientos, no ocasionan impacto significativo para el medio ambiente.

- Si bien la aplicación de la norma trajo impactos positivos para los hoteles y hostales encuestados, el porcentaje de implementación fue considerablemente bajo y se registraron las mismas problemáticas que en el caso Mola Hostel, enfatizando en la falta de recursos financieros, talento humano y conocimientos que permitieran el desarrollo y cumplimiento de la norma.

Finalmente, se puede concluir que la NTS-TS 002 es una norma imprescindible para ayudar a garantizar la sostenibilidad en los establecimientos de alojamiento y hospedaje del país y, aunque su elaboración e implementación para el caso de Mola Hostel tuvo un impacto positivo de gran escala a nivel ambiental, el rendimiento y resultados pueden mejorar si el manual se reestructura o se consideran factores de tipo económico, temporal, espacial (urbano), de recurso humano y de acompañamiento para los pequeños y medianos EAH que se encuentran en el territorio colombiano a la hora de la implementación de la norma. Además, se debe generar un acompañamiento a los EAH que no cuenten con recursos y conocimientos para su elaboración. 


\section{Referencias}

[1] Oficina de Estudios Económicos. "Boletín mensual de turismo", Int. Ministerio de Comercio Industria y Turismo, Colombia, 2017.

[2] E. Rodríguez, (2011, Aug. 10). "Objetivos del turismo sostenible y desarrollo hotelero." Revista el Vinculado [Online]. Available: http://vinculando. org/vacaciones_viajes/turismo_sostenible/turis mo_sostenible_desarrollo_hotelero_objetivos. html\#vcite.

[3] Ministerio de Comercio Industria y Turismo, "Portal MinCIT", MinCIT, Colombia, 2018 [Online]. Available: http://mincit.gov.co/publicaciones/40 284/turismo_en_colombia_crecera_32_en_2018 _segun_el_consejo_mundial_de_viajes_y_turis mo. [Access: 7-Mayo-2019].

[4] Departamento Administrativo Nacional de Estadística, "Muestra mensual de hoteles". Int. DANE, Colombia, COM-030-PD-001-r-004, vol8 2018.

[5] — _ "Clasificación Industrial Internacional Uniforme de todas las actividades económicas." Int. de DANE, Bogotá D.C Colombia. vol 4, pp. 5457. 2012. [Online]. Available: https://www.dane.gov. co/files/nomenclaturas/CIIU_Rev4ac.pdf.

[6] Centro de Información Turística de Colombia, "Estadísticas nacionales- RNT". CITUR, Colombia [Online]. Available: http://www.citur.gov.co/esta disticas/df_prestadores_historico/subCategoria/ 41/P7?t=1 [Access: 10-Junio-2019].

[7] S. Contreras and L. Peñaloza, "El talento humano y el cuidado ambiental en la industria hotelera," Turismo y sociedad, vol. 23, pp. 86-88, 2018. [Online]. Available: https://doi.org/10.18601/ 01207555.n23.05.

[8] E. Vargas, L. Zizumbo, and F. Viesco, "Gestión ambiental en el sector turístico mexicano," Cuadernos de administración, vol. 24, no. 42, pp. 183204, 2011.

[9] A. Kasim, "Towards a winder adoption of environmental responsibility in the hotel sector," International journal of hospitality $\&$ tourism administration, vol. 8, no. 2, pp. 709-725, 2008.

[10] Departamento Administrativo Nacional de Estadística, "Encuentro ambiental de hoteles-EAH 2015", DANE, Bogotá, Colombia, COM-030-PD-001-r004. 2017 [Online]. Available: https://www.dane.gov.co /index.php/estadisticas-por-tema/servicios/encu esta-ambiental-de-hoteles-eah.

[11] G. Fernandez, C. Van, and A. Guzmán, "Alojamientos Turísticos y Medio Ambiente. Diagnóstico Ambiental de los complejos en cabañas en Tandil Argentina," Gestión turística, no. 7, pp. 27-37, 2007.

[12] D. Castro, K. Lopez, and C. Mondragón, "La competitividad del sector hotelero en Colombia en las regiones del Atlántico y el Pacífico," Proyecto de grado. Ciencias Administrativas y Contables. Universidad CES. Santiago de Cali. Cali, Colombia, 2018.

[13] H. Parra, (2014, Jul. 9). "La nueva resolución afecta al sector hotelero". El nuevo día. [Online]. Available: http://www.elnuevodia.com.co/nuevo dia/actualidad/economica/224796-la-nueva-resolu cion-afecta-sector-hotelero.

[14] Instituto Nacional de Turismo, "Estudio: Censo de establecimiento de alojamiento y hospedaje del 2018," Alcaldía Mayor de Bogotá, vol. 7, no. 34, pp. 7-38, 2018.

[15] Congreso de la República, "Ley 1558 de 2012: Por medio de la cual se modifica la Ley 300 de 1996Ley General de Turismo, la ley 1101 de 2006 y se dictan otras disposiciones", Bogotá, Colombia: Diario Oficial, 2012.

[16] Ministerio de Comercio, Industria y Turismo. "Resolución 3860 de 2015: Por la cual se reglamenta el cumplimiento de las Normas Técnicas Sectoriales expedidas por las Unidades Sectoriales de Normalización para las actividades del denominado Turismo de Aventura y la Sostenibilidad Turística”, Bogotá, Colombia: Diario oficial 2015.

[17] M. Aldana, "Análisis de los resultados obtenidos en hoteles de Bogotá, a partir de la implementación de la norma NTS-TS 002," Teoría y paraxis investigativa, vol. 9, no. 2, pp. 36-48, 2014.

[18] C. Sandoval, "Aglomeración turística y de desarrollo económico local, el caso de la localidad de candelaria (Bogotá- Colombia)," Trabajo de grado, Universidad de los Andes, Bogotá, Colombia, 2015.

[19] Centro de actividad regional para la producción más limpia. "Buenas prácticas ambientales en los hoteles". Ministerio de Ambiente España, Cataluña, España. 2006.

[20] X. Medina and D. Zea, "Implementación de una guía metodológica de producción más limpia en el sector hotelero, estudio de caso hotel hacienda Royal". Trabajo de grado. La Salle, Bogotá, 2005.

[21] S. Pieani and H. Arafat, "Solid waste management in the hospitality industry: A review," Journal of environmental management, vol. 146, pp. 320336, 2014.

[22] B. Hoof et al., "Producción más limpia paradigma de la gestión ambiental". Alfa Omega Colombia S.A.S, Bogotá, Colombia, 3B, ISBN: $9789586827-$ $249,2008$.

[23] S. Chan, "Implementing environmental managament System in Small-and Medium-Sized Hotels: Obstacles," Journal of hospitality $\& 3$ tourism research, vol. 35, no. 1, pp. 3-23, 2011.

[24] Secretaría Distrial de Planeación. "Diagnóstico de los aspectos físicos, demográficos y socioeconó- 
micos, conociendo la localidad de Candelaria". Int. Alcaldía Mayor de Bogotá, Bogotá, Colombia. 2009.

[25] Secretaría de Recreación y Deporte. "La Candelaria, observatorio de culturas". Int Alcaldía de Bogotá, Bogotá, Colombia . 2007.

[26] V. Ortiz and H. Olivares, "Diseño de un modelo de gestión ambiental integrado para la implementación de los modelos NTS-TS 002: 2006 y la norma ISO 14001:2004 Aplicada en hoteles de la ciudad de Bogotá,". Tesis de Maestría. Gestión Ambiental. Universidad Javeriana, Bogotá, 2015. 